\title{
Inovação Tecnológica e Desenvolvimento: o Caso da Finlândia
}

\section{Technological Innovation and Development: the Case of Finland}

\author{
Jacqueline Angélica Haffner* \\ Ariane Bayer de Oliveira**
}

\begin{abstract}
Resumo: O presente artigo visa analisar as principais transformações econômicas ocorridas na Finlândia desde a recessão que atingiu o país em 1990, dando especial atenção às políticas econômicas de fomento às inovações tecnológicas, que possibilitaram a superação da crise e a promoção de um crescimento econômico marcado por uma alta competitividade econômica e pela manutenção do bem-estar da população. Para isso, o artigo investiga a formulação de uma estratégia nacional de inovação, direcionada à mobilização de diversos atores econômicos na construção de um sistema nacional de inovação finlandês dinâmico e capaz de alavancar o desenvolvimento de uma economia do conhecimento finlandesa.
\end{abstract}

Palavras-chave: Inovações tecnológicas. Crescimento econômico. Pesquisa e desenvolvimento.

\begin{abstract}
This paper aims at analyzing key economic transformations of Finland since an economic recession hit the country in 1990, focusing on the main economic policies used to foster technological innovations and that allowed a recovery from the crisis, as well as promoted a highly competitive and welfare related economic growth. The paper also analyses the formulation of a national innovation strategy designed to mobilize several economic actors in the making of a dynamic Finnish National Innovation System that is able to leverage the development of a Finnish Knowledge Economy.
\end{abstract}

Keywords: Technological innovation. Economic growth. Research e development. JEL Classification: $\mathrm{O} 33$.

\section{1 lntrodução}

A aptidão finlandesa para a adoção de alternativas autônomas de crescimento incorporando inovações produtivas deriva de sua trajetória histórica de crescimento desde meados do século XIX. Contudo, a década de 1990 propiciou um marco histórico no desenvolvimento do país após uma profunda recessão obrigá-lo a encarar uma postura inovadora mais ativa e pioneira, capaz de produzir transformações estruturais significativas (KIRBY, 2009, p. 289; KLINGE, 2000, p.

* Professora do Departamento de Economia e Relações Internacionais e do Programa de Pós-graduação em Estudos Estratégicos Internacionais da Universidade Federal do Rio Grande do Sul (UFRGS). E-mail: jacqueline.haffner@ufrgs.br

* $\quad$ Bacharela em Relações Internacionais pela Universidade Federal do Rio Grande do Sul (UFRGS). E-mail: aryanabayer@hotmail.com 
145-165). Parte dessa transformação centrou-se na formulação de uma estratégia de construção de uma economia do conhecimento, baseada na visão sistêmica do sistema nacional de inovação finlandês (Snif), fonte e alvo de investimentos intensivos em atividades de pesquisa e desenvolvimento (PED).

O setor núcleo desse sistema, principal motor da economia desde meados da década de 1990, constituiu-se de uma moderna indústria de tecnologia da informação e comunicação (TIC), da qual o principal instrumento dinâmico era a empresa finlandesa Nokia. As reestruturações alavancadas pela depressão conseguiram ampliar as opções produtivas e, não somente permitiram a superação da crise, como também tornaram a Finlândia um dos países mais desenvolvidos do mundo, exemplo de economia do conhecimento e líder de rankings de competitividade internacional, educação e qualidade de vida.

\section{A Crise Econômica de 1990}

A trajetória de crescimento econômico da Finlândia pós-Segunda Guerra tornou-lhe uma das nações que mais progridem no mundo (SINGLETON, 1998, p. 153). Essa prosperidade perdurou até o fim da década de 1980, quando o país experimentou um boom econômico. As taxas de desemprego estavam abaixo da média dos países da OCDE, as taxas de crescimento figuravam acima da média europeia, não havia dívida externa ou pública significativas e o desempenho macroeconômico do país era positivo (HONKAPOHJA et al., 2009, p. 4-10; KIANDER, 2004, p. 3; KIRBY, 2009, p. 291).

Durante a década de 1980, a Finlândia entrou na fase de desregulação e liberalização financeira que se disseminava pelo sistema internacional e eliminou restrições a empréstimos externos e controles sobre as taxas de juros, mantendo apenas um regime cambial fixo.

Nos anos de 1985-1986, o aprofundamento da desregulação dos mercados financeiros e a valorização da moeda nacional - o markka - estimularam a explosão de crédito e de empréstimos externos e provocaram a entrada e a saída excessivas de capitais, impelindo a economia a uma fase de superaquecimento econômico (HONKAPOHJA et al., 2009, p. 8; JONUNG; KIANDER; VARTIA, 2008, p. 13-14; JONUNG; SÖDERSTRÖM; STYMNE, 1996, p. 56; YLÄ-ANTTILA; LEMOLA, 2003, p. 5). Fatores externos, tais como o aumento internacional dos termos de troca provocado pela queda no preço de recursos energéticos e pela simultânea elevação dos preços de produtos florestais, aliaram-se à liberalização do fluxo de capitais no fomento do boom econômico (KIANDER, 2004, p. 4-5).

Em âmbito doméstico, as políticas econômicas conduzidas pelo governo finlandês até 1989 não eram suficientemente restritivas para combater tal situação. A política fiscal expansionista adotada incentivou o consumo e os investimentos 
públicos, contribuindo para a aceleração da economia e para o incremento do déficit público. A política monetária restritiva, por seu lado, conjugada a um regime cambial fixo valorizado e às altas taxas de juros, incrementou o fluxo de capitais (JONUNG; KIANDER; VARTIA, 2008, p. 14-16; KIANDER, 2004, p. 6-7).

A expansão econômica súbita reduziu o desemprego de 4\%, em 1987, para 2,5\%, ao fim de 1989, e elevou a inflação de 2\%, em 1986, para 7\%, em 1989. Esse processo pressionou a taxa de juros e causou o robustecimento da demanda agregada, a qual propagou desequilíbrios na balança externa e, consequentemente, na conta corrente do país (HONKAPOHJA et al., 2009, p. 11). A concessão abundante de crédito, por sua vez, expandiu a dívida do setor privado e dobrou os preços de ativos e bens imobiliários durante o boom (HONKAPOHJA et al., 2009, p. 22; JONUNG; SÖDERSTRÖM; STYMNE, 1996, p. 56, 62-63).

Em 1990, a política econômica inapropriada, aliada a choques externos, mostrou-se insustentável, e a Finlândia mergulhou na pior recessão da história do país, superando a depressão de 1930. A política fiscal expansionista, e apenas parcialmente contracíclica, foi substituída por uma política restritiva (contracionista) entre 1991 e 1994, a qual se focou na desaceleração da economia através do aumento de impostos e da redução de gastos públicos, a fim de contrair o consumo e a inflação (JONUNG; KIANDER; VARTIA, 2008, p. 22-24).

Por fim, o câmbio fixo e ajustável, valorizado artificialmente em 1989, foi abandonado e a moeda nacional desvalorizou-se em 12\% em novembro de 1991. Em setembro de 1992, adotou-se o câmbio flutuante e as exportações voltaram a crescer (HONKAPOHJA et al., 2009, p. 42; JONUNG; SÖDERSTRÖM; STYMNE, 1996, p. 66; KIANDER, 2004, p. 10; KIANDER; VARTIA, 1996, p. 76-78). Nesse contexto, a elevação da taxa de juros real, a redução da inflação e a subsequente depreciação do markka provocaram queda substancial de todos os componentes domésticos da demanda agregada, com destaque para os investimentos e o consumo privados e públicos, conforme demonstrado nos Gráficos 1 e 2 (HONKAPOHJA et al., 2009, p. 16-17; HONKAPOHJA; KOSKELA; PAUNIO, 1996, p. 39; JONUNG; SÖDERSTRÖM; STYMNE, 1996, p. 56-57; KIANDER; WIDGRÉN, 1996, p. 3). 
Gráfico 1 - Efeitos da crise de 1990 no consumo privado (1985-2008), em milhões de marcos finlandeses (FIM)

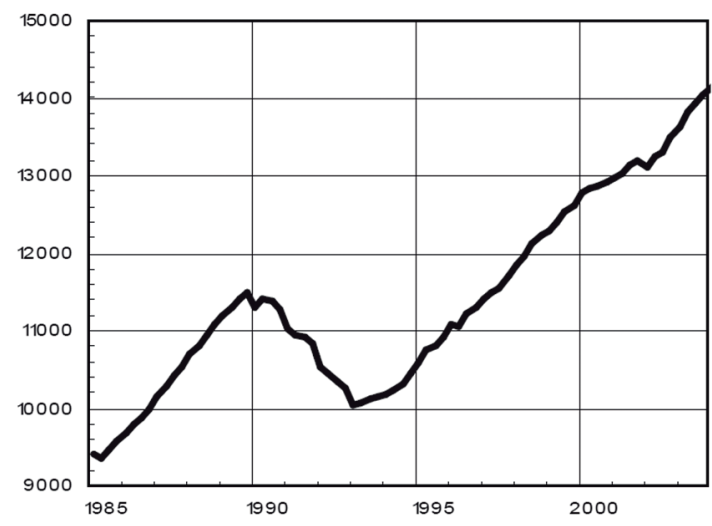

Fonte: Honkapohja et al. (2009).

Gráfico 2 - Efeitos da crise de 1990 nos investimentos privados (1985-2008), em milhões de marcos finlandeses (FIM)

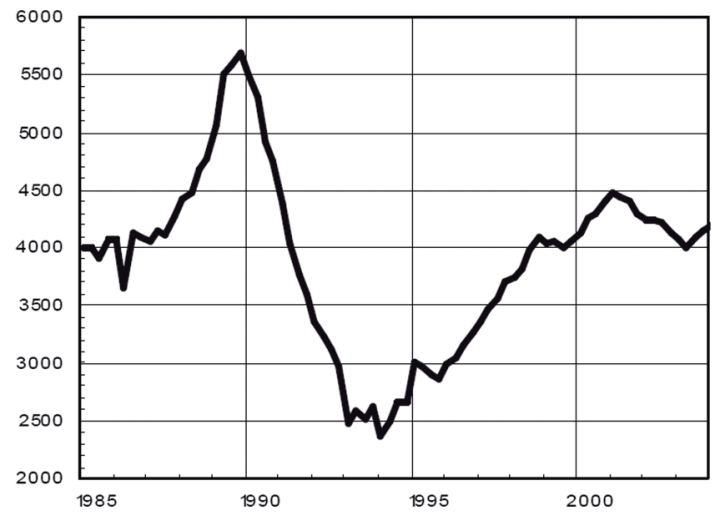

Fonte: Honkapohja et al. (2009).

O declínio do consumo e dos investimentos públicos, no entanto, não evitaram um salto da dívida pública de menos de 10\% do PIB, em 1990, para 75\% a partir de 1991 (KIRBY, 2009, p. 292). O aumento da dívida foi causado, principalmente, pela baixa carga tributária, pelos altos custos sociais gerados pela massa crescente de desempregados e pela redução do PIB (UUSITALO, 1996 e 2000).

A queda do PIB real alcançou 14\% entre 1990 e 1993, e a taxa de crescimento do PIB real, que era de 5,4\%, em 1989, desmoronou para 6,5\% negativos, em 1991, continuando a decair lentamente até o ponto de inflexão da fase recessiva, 
em 1993 (ver Gráfico 3) (HONKAPOHJA et al., 2009, p. 3, 16-17; JONUNG; SÖDERSTRÖM; STYMNE, 1996, p. 56; KIANDER; VARTIA, 1996, p. 74; KIANDER; WIDGRÉN, 1996, p. 3). Os efeitos sociais da depressão incluíram aumento significativo do desemprego, que passou de menos de 3\%, em 1990, para quase $20 \%$ no início de 1994 (ver Gráfico 4). O número total de empregos aniquilados durante os quatro anos da crise (1990-1994) chegou a 450.000. Em longo prazo, as mudanças estruturais provocadas pela crise deram origem a um desemprego estrutural que ainda persiste (ERVASTI, 2002; GRÖNQVIST; KINNUNEN, 2009; VERHO, 2008).

Gráfico 3 - Queda no crescimento do PIB real (1980-2008)

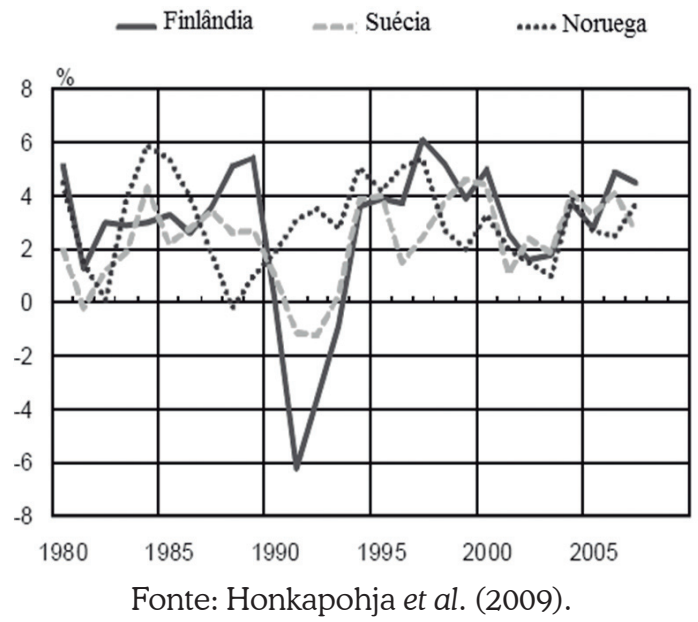

Gráfico 4 - Desemprego (1980-2008)

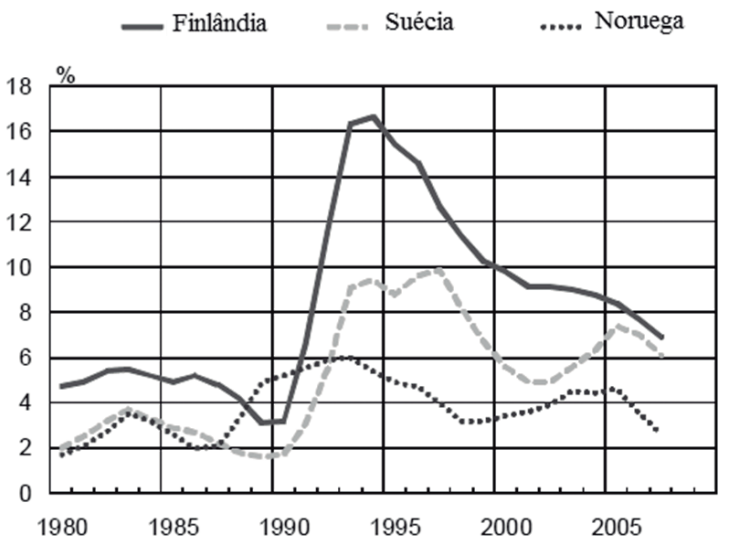

Fonte: Honkapohja et al. (2009). 
O superaquecimento e a depressão econômica também provocaram o colapso do sistema bancário e a falência de diversas empresas tomadoras de crédito (HONKAPOHJA; KOSKELA; PAUNIO, 1996, p. 40; JONUNG; SÖDERSTRÖM; STYMNE, 1996, p. 65). A superação de tal declínio só aconteceria em 1998, após intervenções governamentais e parlamentares instituírem intensa reestruturação do sistema bancário (HONKAPOHJA et al., 2009, p. 12, 16, 20-24; KIANDER; WIDGRÉN, 1996, p. 3).

As causas para a eclosão da recessão econômica finlandesa de 1990 derivam de diversos fatores (HONKAPOHJA; KOSKELA; PAUNIO, 1996, p. 38; YLÄ-ANTTILA; LEMOLA, 2003, p. 2). Primeiramente, as deficiências na implantação da liberalização financeira no país, aliadas a políticas macroeconômicas ineficientes no combate a uma situação de superaquecimento, criaram o cenário doméstico instável (HONKAPOHJA et al., 2009, p. 17-20; HONKAPOHJA; KOSKELA; PAUNIO, 1996, p. 46; JONUNG; SÖDERSTRÖM; STYMNE, 1996, p. 64-65; KIANDER; VARTIA, 1996, p. 73, 82-83; ORNSTON; REHN, 2006, p. 10). Além disso, a alta nas taxas de juros finlandesas, impulsionada pela reunificação alemã e pela defesa do markka de ataques especulativos por meio de alta nas taxas de juros nominais, completou o rol de fatores domésticos que moldaram a crise (HONKAPOHJA et al., 2009, p. 17-19; JONUNG; SÖDERSTRÖM; STYMNE, 1996, p. 64; KIANDER; VARTIA, 1996, p. 75-76).

O cenário internacional também colaborou para que a fase de superaquecimento econômico no fim da década de 1980 se tornasse um trem desgovernado (DAHLMAN; ROUTTI; YLÄ-ANTTILA, 2007, p. 3; HONKAPOHJA et al., 2009, p. 11; JONUNG; KIANDER; VARTIA, 2008, p. 22; KIANDER; VARTIA, 1996, p. 72-74; KLINGE, 2000, p. 162, 164; KIRBY, 2009, p. 291). A desaceleração do crescimento econômico internacional reduziu as exportações finlandesas. Somado a isso, o aumento nos preços internacionais de derivados florestais deteriorou os termos de troca e reduziu sua vantagem competitiva no mercado internacional (ver Gráfico 5). Finalmente, o colapso da União Soviética, em 1991, eliminou 70\% das trocas entre os dois países, ocasionando 3\% de queda no PIB finlandês (ORNSTON, REHN, 2006, p. 1). 


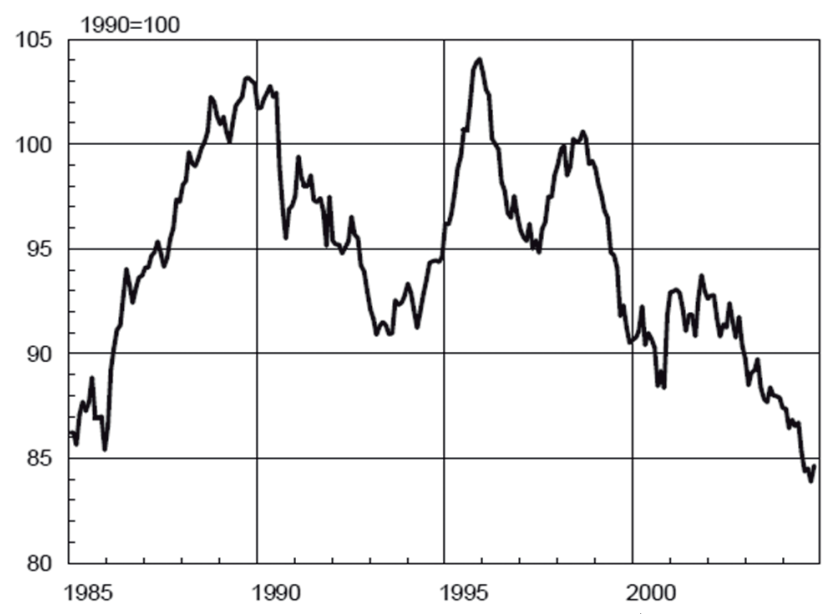

Fonte: Honkapohja et al. (2009).

Na metade da década de 1990, a Terra dos Lagos protagonizou uma rápida transição do caos econômico para um crescimento acelerado de 4,5\% anual, com taxas de desemprego de 9\%, em 2001, e ganhos crescentes de produtividade (KIANDER, 2004, p. 11; WERNER, 2003, p. 1-4). Esses dados foram resultado da construção de um novo ciclo produtivo baseado na geração de uma nova economia (DAVERI, 2002) centrada na produção de conhecimento e de inovações tecnológicas. A próxima sessão deste artigo se destina à análise do processo supracitado, destacando os passos tomados na construção de uma estratégia inovadora.

\section{A Estratégia Nacional de Inovação}

A rápida ascensão finlandesa da grave depressão iniciou-se com a adoção de políticas macroeconômicas reparadoras (KIANDER, 2004, p. 16). Nesse sentido, o abandono do regime cambial fixo, a política monetária voltada para o combate à inflação e a política de consolidação fiscal como parte de uma estratégia de adequação às exigências da União Europeia auxiliaram na recuperação imediata da decadência econômica a tempo de permitir a inclusão do país no bloco europeu em 1995 (BOSCHMA; SOTARAUTA, 2007, p. 163; HONKAPOHJA et al., 2009, p. 41-53; JONUNG; KIANDER; VARTIA, 2008, p. 31; JONUNG; SÖDERSTRÖM; STYMNE, 1996, p. 69; KIANDER; VARTIA, 1996, p. 76-78; KIRBY, 2009, p. 293; KLINGE, 2000, p. 163).

Na primeira metade da década, a Finlândia retomou rapidamente o caminho para o desenvolvimento, reduzindo a dívida pública, assegurando a credibilidade 
fiscal, retomando as exportações e reestruturando o sistema bancário sem gerar desequilíbrios no setor público devido ao aumento dos tributos e aos cortes de gastos governamentais (JONUNG; KIANDER; VARTIA, 2008, p. 31; KIANDER; VARTIA, 1996, p. 78-79; KIANDER; WIDGRÉN, 1996, p. 3). Essa retomada do crescimento deu-se, em parte, por causa da reestruturação schumpeteriana da economia, característica da flutuação natural dos ciclos produtivos (ANDERSEN et al., 2006; BOSCHMA; SOTARAUTA, 2007; MALIRANTA; ROUVINEN; YLÄANTTILA, 2010; SCHIENSTOCK, 2007; YLÄ-ANTTILA; LEMOLA, 2003, p. 14). Ou seja, por causa do aprofundamento do processo de destruição criadora, iniciado em 1980, a realocação de recursos desativados por ocasião da crise permitiu que empresas e setores mais eficientes tomassem a dianteira da economia (HONKAPOHJA et al., 2009, p. 57-62; JONUNG; KIANDER; VARTIA, 2008, p. 33; KAUTTO-KOIVULA et al., 2008, p. 7; KIANDER, 2004, p. 14; SCHIENSTOCK, 2007).

Na década de 1980, a orientação tecnológica dada à economia aprofundou o comprometimento com a construção de uma economia do conhecimento (BOSCHMA; SOTARAUTA, 2007, p. 167; JAUHIAINEN, 2009, p. 12-14; MALIRANTA; ROUVINEN; YLÄ-ANTTILA, 2010, p. 82; OINAS, 2005, p. 1234-1235; ORNSTON; REHN, 2006, p. 17; WERNER, 2003, p. 4-8; YLÄ-ANTTILA; LEMOLA, 2003, p. 3 , 9). Contudo, apenas na década de 1990 as transformações no sistema internacional e a reestruturação econômica forçada pela crise incutiram nas autoridades governamentais a percepção de que o crescimento econômico acelerado não seria mais impulsionado pelos setores industriais tradicionais, mas sim por uma nova opção produtiva (JONUNG; KIANDER; VARTIA, 2008, p. 32-33; ORNSTON; REHN, 2006, p. 10). A partir de então, a construção de uma economia das ideias (POWELL; SNELLMAN, 2004) passou a ser profundamente propagada e perseguida por toda a nação por meio da ampliação intensiva da capacidade inovadora e tecnológica nacional (KIANDER, 2004, p. 12; SCHIENSTOCK, 2007, p. 104-105; YLÄ-ANTTILA; LEMOLA, 2003, p. 8).

As condições externas favoráveis devido ao boom do setor de TIC (KIANDER, 2004, p. 12; YLÄ-ANTTILA, LEMOLA, 2003, p. 2) e a reestruturação do mercado financeiro após a crise de 1990 possibilitaram o máximo aproveitamento da reestruturação finlandesa e, por conseguinte, do crescimento econômico (JAUHIAINEN, 2009, p. 14-15). Isso porque a ampliação dos mecanismos de crédito e poupança e o aumento de eficiência na alocação de recursos em direção à produção inovadora e no controle de governança criaram um espaço de comercialização das novas tecnologias desenvolvidas (DAHLMAN; ROUTTI; YLÄ-ANTTILA, 2007, p. 47; SCHIENSTOCK, 2007, p. 110). Além disso, a existência de um consenso nacional sobre a necessidade de transformação econômica e social propiciou o momentum ideal para a incursão numa nova trajetória desenvolvimentista (KIANDER, 2004, p. 8; TOLEDO; ARBIX; SALERNO, 2009, p. 7, 11). 
Por fim, em 1993 e 1994, os discursos americanos sobre a importância da indústria informativa na economia e a formulação do Relatório Bangemann da União Europeia, a fim de instituir estratégias de tecnologia da informação no bloco, estabeleceram as diretrizes para a formulação de uma estratégia nacional finlandesa de inovação (JAUHIAINEN, 2009, p. 16; ORNSTON; REHN, 2006, p. 21, 27; WERNER, 2003, p. 11). Com isso, o país passou a criar condições para as transformações estruturais necessárias às atividades de inovação, com ênfase na tecnologia, na construção de uma economia do conhecimento e no incremento da produtividade, a fim de impelir o desenvolvimento econômico nacional (DAHLMAN; ROUTTI; YLÄ-ANTTILA, 2007, p. 53-55; JONUNG; KIANDER; VARTIA, 2008, p. 33). Nesse contexto, as inovações eram vistas como motor do crescimento econômico e como base para obtenção de competitividade e de oportunidades econômicas. Por meio dessas, o conhecimento seria aplicado de maneira mais eficiente a fim de dinamizar a economia (FURMAN; PORTER; STERN, 2001; JAUHIAINEN, 2009, p. 6; TOLEDO; ARBIX; SALERNO, 2009, p. 8-9).

Os primeiros passos efetivos na construção de uma estratégia nacional derivam da formulação de uma ideologia inovadora pelo Conselho de Ciência e Tecnologia no início da década de 1990. Tal corpo de princípios centrava-se nos conceitos de sistema nacional de inovação (SNI) e de economia do conhecimento como base para a formulação de políticas tecnológicas, aplicados pela primeira vez no mundo (BOSCHMA; SOTARAUTA, 2007, p. 168; YLÄ-ANTTILA; LEMOLA, 2003, p. 7). O Conselho também destacou a importância de um sistema de PEBD, de um sistema educacional de qualidade e de um ambiente aberto ao desenvolvimento e à aplicação de novas tecnologias, bem como da cooperação nacional e internacional na formação do SNI (JAUHIAINEN, 2009, p. 14-16; YLÄ-ANTTILA; LEMOLA, 2003, p. 9).

Novos esforços sucederam em 1994, quando o Relatório Tikas (KIRBY, 2009) produzido no governo de Esko Aho (1991-1995) com o objetivo de formular políticas contemporâneas para a formação de uma sociedade do conhecimento, explicitou a adoção de uma trajetória econômica embasada na produção e no uso de TIC. Um ano antes (1993), o Ministério do Comércio e da Indústria publicou um relatório chamado Documento branco para estratégia industrial nacional (FINLÂNDIA, 1993), que criou as diretrizes para a reestruturação econômica através da constituição de uma nova economia da Finlândia (JONUNG; KIANDER; VARTIA, 2008, p. 33).

O Documento branco para estratégia industrial nacional também incitava a construção de novas políticas industriais centradas no fortalecimento dos conglomerados industriais a fim de promover a formação de um Snif (DAHLMAN; ROUTTI; YLÄ-ANTTILA, 2007, p. 39), embasado na indústria de TIC como futuro baluarte econômico. Essas novas políticas industriais atuariam para promover o 
crescimento industrial por meio de um ambiente propício a inovações. É importante ressaltar que o conceito de nova economia abarca o uso de avançadas tecnologias de conhecimento, especialmente de TIC e biotecnologia, na geração de uma forma de produção inovadora (DAHLMAN; ROUTTI; YLÄ-ANTTILA, p. 40, 2007; HONKAPOHJA et al., 2009, p. 89).

As políticas industriais estabelecidas pelo documento estariam centradas no conceito de conglomerados industriais, devido à crença de que indústrias e empresas possuem crescente interdependência, com trocas constantes de informações, spillovers tecnológicos e inovadores e formação de cadeias produtivas (JAUHIAINEN, 2009, p. 16; ORNSTON; REHN, 2006, p. 18; TOLEDO; ARBIX; SALERNO, 2009, p. 10; WERNER, 2003, p. 11; YLÄ-ANTTILA; LEMOLA, 2003, p. 7). Nesse contexto, as políticas industriais centradas na manutenção da cooperação entre indústrias e empresas seriam mais eficientes para assegurar a especialização e impulsionar ondas de inovação na economia.

Para concretizar tal projeto, o documento estabelecia como objetivos norteadores a geração de condições para o progresso de fatores de produção, a ampliação da competitividade econômica por meio do aumento dos gastos públicos em PẺD, a educação, a infraestrutura tecnológica e a atuação governamental como coordenadora das políticas industriais e como provedora de informação entre as partes da rede nacional de inovação (YLÄ-ANTTILA; LEMOLA, 2003). Desde então, a Finlândia adotou o comprometimento em longo prazo com a aplicação de políticas públicas responsáveis que originassem o ambiente inovador entre conglomerados industriais, organizações condutoras de PED, federações industriais, empresas e universidades (JAUHIAINEN, 2009, p. 21; MALIRANTA; ROUVINEN; YLÄ-ANTTILA, 2010, p. 82; OINAS, 2005, p. 1235; YLÄ-ANTTILA; PALMBERG, 2007).

A construção de uma estratégia nacional, entretanto, só foi formulada após o Ministério do Emprego e Economia (até o fim de 2007, Ministério do Comércio e da Indústria) indicar um grupo de direção na preparação de uma estratégia nacional de inovação finlandesa, dentro do programa do segundo gabinete do primeiro-ministro Matti Vanhanen (FINLÂNDIA, 2008; JAUHIAINEN, 2009, p. 20). Em 2008, a proposta foi aceita pelo governo, que passou a delinear as diretrizes de uma Política Nacional de Inovação, a ser conduzida no sentido de impulsionar o desenvolvimento do ambiente inovador finlandês, o crescimento econômico, o bem-estar social e a competitividade nacional. A Estratégia Nacional de Inovação, desenvolvida após a crise de 2008, também aborda os desafios impostos ao sistema produtivo finlandês, bem como maneiras de enfrentá-los com eficiência, revertendo, ou pelo menos evitando, o declínio da competitividade (FINLÂNDIA, 2008; OINAS, 2005, p. 1239-1240).

A criação desse ambiente inovador, buscado pelo setor público com afinco desde a publicação do Documento branco, tornou-se uma responsabilidade con- 
junta de empresas e indústrias, que incentivam e financiam atividades de PEBD e concretizam as novas descobertas tecnológicas: de universidades, que fomentam a atividade de PED, além de formarem capital humano; e da sociedade, que adota a educação contínua como forma de qualificação profissional e que satisfaz a demanda de mão de obra qualificada. Esse conjunto de atores, por sua vez, passou a constituir o denominado Snif (DAHLMAN; ROUTTI; YLÄ-ANTTILA, 2007, p. 41; OINAS, 2005, p. 1235).

\section{O Sistema Nacional de Inovação Finlandês}

O Snif foi criado em 1990 pelo Conselho Nacional de Política para Ciência e Tecnologia, atualmente Conselho para Pesquisa e Inovação, como uma estrutura fundamental, que estimula a coordenação entre um conjunto de atores incumbidos de promover o crescimento econômico dentro de regras de difusão do conhecimento e da tecnologia (ARBIX; FERRAZ, 2009, p. 230; BLOMSTRÖM; KOKKO; SJÖHOLM, 2002, p. 16; OINAS, 2005, p. 1235-1236; SCHIENSTOCK, 2007, p. 111; WERNER, 2003, p. 9; YLÄ-ANTTILA; LEMOLA, 2003, p. 7). O SNI é coordenado diretamente pelo parlamento, o qual determina o nível de recursos disponíveis às atividades de $\mathrm{P} \mathcal{} 3 \mathrm{D}$ e às novas combinações produtivas. Em âmbito público, o governo atua através do Comitê para o Futuro, do Conselho para Pesquisa e Inovação, do Ministério da Educação e da Cultura e do Ministério do Emprego e da Economia (resultado da fusão do Ministério do Comércio e da Indústria e do Ministério do Trabalho), entre outros, a fim de eliminar diferenciações entre política industrial e política tecnológica e científica (DAHLMAN; ROUTTI; YLÄ-ANTTILA, 2007, p. 41; FINNISH SCIENCE AND TECHNOLOGY INFORMATION SERVICE, 2011; JAUHIAINEN, 2009, p.19-20; SCHIENSTOCK, 2007, p. 112).

O Comitê para o Futuro, criado em caráter temporário em 1993 e tornado permanente em 2000, destina-se a coordenar o diálogo com o parlamento a respeito das necessidades aparentes na construção de uma economia do conhecimento (DAHLMAN; ROUTTI; YLÄ-ANTTILA, 2007, p. 38; PARLAMENTO DA FINLÂNDIA, 2011). O Conselho para Pesquisa e Inovação, originalmente apenas Conselho de Políticas para Ciência (1963), é um órgão de conselho governamental encabeçado pelo primeiro-ministro finlandês e com o objetivo de promover a integração entre os setores tecnológico, científico e econômico (JAUHIAINEN, 2009, p. 19; MINISTÉRIO DA EDUCAÇÃO E DA CULTURA, 2011; WERNER, 2003, p. 10). Desde 2003, o Conselho para Pesquisa e Inovação também passou a abordar questões sociais e regionais para a eficiência das políticas nacionais de inovação.

O Ministério da Educação e da Cultura e o Ministério da Indústria e Comércio estão diretamente ligados à geração e à coordenação de condições técnicas e econômicas para a perpetuação de políticas tecnológicas e científicas (DAHLMAN; 
ROUTTI; YLÄ-ANTTILA, 2007, p. 43). Enquanto o primeiro coordena a Política Nacional de Ciências e administra a Academia da Finlândia, o segundo é responsável pela política de tecnologia, através da Agência de Tecnologia e Inovação Nacional (Tekes) e pelo Centro de Pesquisa Técnica da Finlândia (VTT).

A Academia da Finlândia, a Tekes e o Fundo Nacional Finlandês para Pesquisa e Desenvolvimento (Sitra) são os órgãos do sistema responsáveis pela administração do financiamento da atividade básica ou aplicada de P\&D, bem como pelo desenvolvimento de projetos e pelo fornecimento de conhecimento (ACADEMY OF FINLAND, 2011; JAUHIAINEN, 2009, p. 20; SITRA, 2011; TEKES, 2011). A Academia da Finlândia em seu formato final de 1970, subordinada ao Ministério da Educação e da Cultura, é o principal meio de alocação de recursos para a pesquisa científica básica (ACADEMY OF FINLAND, 2011; DAHLMAN; ROUTTI; YLÄ-ANTTILA, 2007, p. 59).

A Tekes subordina-se ao Ministério do Emprego e da Economia e foi criada em 1983 para formular políticas modernizadoras através do financiamento de programas de pesquisa em empresas, instituições e universidades (TEKES, 2011). Em 2010, os investimentos provenientes da agência alcançaram 633 milhões de euros, dos quais grande parte foi destinada a pequenas e médias empresas (DAHLMAN; ROUTTI; YLÄ-ANTTILA, 2007, p. 44; PALMBERG et al., 2000; TEKES, 2011).

O Sitra, órgão de excelência originado em 1961, mas operando desde 1991 sob autoridade do parlamento finlandês, possui renda atual de 500 milhões de euros, destinados à condução de políticas econômicas voltadas ao fomento de atividades inovadoras pioneiras, que impulsionam o desenvolvimento da economia da informação (DAHLMAN; ROUTTI; YLÄ-ANTTILA, 2007, p. 36; JAUHIAINEN, 2009, p. 22; SITRA, 2011). A Academia da Finlândia e a Tekes são inteiramente financiadas pelo setor público, enquanto o Sitra conta com capital privado.

As 16 universidades, 27 politécnicas e 21 institutos de pesquisa (MINISTÉRIO DA EDUCAÇÃO E DA CULTURA, 2011; STUDY IN FINLAND, 2011) - entre os quais se inclui o VTT - trabalham como operadores do sistema, levando a cabo a atividade de pesquisa efetiva. As principais universidades constituintes do núcleo de pesquisa finlandês são a Universidade de Helsinki, a de Jyväaskylä, a de Oulu e a Universidade de Tecnologia de Helsinki e a de Tampere. O VTT, estabelecido em 1942, é um ator vital do SNI e é a maior organização de pesquisa politécnica aplicada do Norte da Europa. Sua principal função é desenvolver novas tecnologias aplicadas e fortalecer as redes de cooperação tecnológica nacional e internacionalmente (DAHLMAN; ROUTTI; YLÄ-ANTTILA, 2007, p. 47; JAUHIAINEN, 2009, p. 20; VTT, 2011).

Outros atores do Snif, tais como os centros de especialização e os centros de desenvolvimento econômico, transporte e meio ambiente agem como coordenadores de políticas que assegurem a competitividade de suas respectivas esferas de influência. O programa de centros de especialização, lançado em fases (1994-1998, 
1999-2006, 2007-2013), cria um ambiente de interação local e impulsiona a competitividade de cada região (JAUHIAINEN, 2009, p. 25-29). Os centros de desenvolvimento econômico, transporte e meio ambiente, por sua vez, um total de 15 ao redor do país, dão apoio às pequenas e médias empresas e fortalecem as redes de cooperação regionais (DAHLMAN; ROUTTI; YLÄ-ANTTILA, 2007, p. 45; MINISTÉRIO DO EMPREGO E DA ECONOMIA, 2011).

Instituições diversas, como a Finpro e Finnvera, aumentam o escopo de atuação do Snif, pois favorecem a internacionalização e oferecem crédito à exportação das empresas finlandesas, respectivamente (FINNVERA, 2011; FINPRO, 2011). Além dessas, a Fundação para Invenções Finlandesas dá apoio a novas propostas inovadoras (INNOFIN, 2011), e a Finnish Industry Investment Ltd, uma companhia estatal, estimula o venture capital por meio de seus investimentos nas empresas do Snif (INDUSTRY INVESMENT, 2011).

O setor privado completa o Snif e detém a maior importância relativa deste, porque inclui não só empresas que virtualmente empregam as inovações, mas também institutos de pesquisa, fundações privadas e sociedades acadêmicas e industriais que incrementam os investimentos em PED, ampliam a coordenação intra e intersetoriais e impulsionam a nova economia da Finlândia. O comprometimento crescente do setor privado com a trajetória inovadora revela-se nos altos níveis de emprego de novas combinações produtivas e organizacionais nas empresas finlandesas (ARCHIBUGI; DENNI; FILIPPETTI, 2009, EUROPEAN COMMISSION, 2006, 2007, 2008, 2009, 2010; EUROSTAT EUROPEAN COMMISSION, 2007a, 2008a, 2009). Entre 2006 e 2008, a média de empresas que adotaram modernizações foi de $47 \%$, das quais $31 \%$ investiram em novos produtos, $34 \%$ em novos processos e $25 \%$ em projetos de inovação (FINNISH SCIENCE AND TECHNOLOGY INFORMATION SERVICE, 2011; PARVAN, 2007).

O grau de profusão das inovações tecnológicas no SNI se deve, em grande parte, à importância dada à atividade de PEBD pelos setores público e privado. Desde as origens da formação da nova economia finlandesa, investimentos em atividades de PEBD são relacionados a ganhos de produtividade e, portanto, ao crescimento econômico. Ou seja, não existiram inovações sem PẺD e, na mesma lógica, não existiria um sistema de inovação sem inovações. Devido a isso, a próxima sessão do artigo enfocará o desenvolvimento da atividade de PEB na Finlândia e sua comparação em termos internacionais.

\section{Pesquisa e Desenvolvimento}

A transição finlandesa para uma economia do conhecimento especializada em TIC se deve a dois fatores-chave: os investimentos em PEBD e o comprometimento com a educação de qualidade (BOSCHMA; SOTARAUTA, 2007, p. 164; 
JONUNG; KIANDER; VARTIA, 2008, p. 33; SCHIENSTOCK, 2007, p. 111). A intensidade de PEBD é uma medida das inovações de um país e, conjugadas, impulsionam a economia nacional e o bem-estar social, disseminando a prosperidade por toda a nação (CZARNITZKI; EBERSBERGER; FIER, 2004; HONKAPOHJA et al., 2009, p. 90). Na Finlândia, o financiamento de PEBD preza pela cooperação e pela competitividade de acordo com as diretrizes do Snif (ORNSTON; REHN, 2006, p. 18; WERNER, 2003, p. 8). Nesse sentido, a cooperação é assegurada através da liberação de reservas para atividades de pesquisa apenas às empresas conectadas a outros atores do Snif (DAHLMAN; ROUTTI; YLÄ-ANTTILA, 2007, p. 48).

Devido a isso, a Finlândia tem se estabelecido entre os líderes mundiais de colaboração. Em 2003, o país foi líder em colaboração entre atores do SNI no ranking da União Europeia de Inovação (EUROPEAN COMMISSION, 2003). Em 2004, a Community Innovation Statistics (CIS 2004) da Eurostat revelou que 58\% das empresas finlandesas colaboraram, abaixo apenas da média de Chipre (68\%) (DAHLMAN; ROUTTI; YLÄ-ANTTILA, 2007, p. 48; PARVAN, 2007). Em razão da crise de 2008, os índices finlandeses sofreram estabilização e as empresas inovadoras decaíram para pouco mais de 50\%, dentro da média da União Europeia (EUROPEAN COMMISSION, 2008, 2009, 2010, 2011).

Desde 1990, a participação pública no financiamento de atividades de PEBD decaiu significativamente para 24\%, em 2009 (EUROSTAT EUROPEAN COMMISSION, 2007a, 2008a, 2009), abaixo da média da União Europeia de 34\%. Apesar da queda relativa, os investimentos públicos absolutos cresceram durante a crise de 1990 e atualmente se mantêm acima da média da União Europeia. Em 2008, enquanto os governos europeus destinavam 0,72\% do PIB à PEBD, na Finlândia os gastos governamentais chegaram a quase $1 \%$ do PIB, atrás apenas da Espanha $(1,07 \%)$ e de Portugal (1,02\%) (EUROSTAT EUROPEAN COMMISSION, 2008, 2009, 2010a, 2010b, 2011a, 2011b).

Mesmo com a queda na participação governamental nos investimentos tecnológicos, o total de inversões públicas e privadas em PEBD na Finlândia cresceu e, desde 1994, excede as médias da União Europeia e da OCDE (MALIRANTA; ROUVINEN; YLÄ-ANTTILA, 2010, p. 82; ORGANISATION FOR ECONOMIC CO-OPERATION AND DEVELOPMENT, 2002, ORNSTON; REHN, 2006, p. 8; WERNER, 2003, p. 6-7). Em termos numéricos, os gastos anuais totais nas atividades de pesquisa representaram 3,5\% do PIB, em 2004. Em 2009, essa porcentagem alcançou 3,96\% do PIB e, em 2010, 3,88\%, acima da média da União Europeia de 1,85\% (EUROSTAT EUROPEAN COMMISSION, 2007b, 2009).

Tal índice incorpora o incremento da participação do setor privado, que é o grande responsável atual pela intensidade em PED finlandesa (EUROPEAN COMMISSION, 2003, 2011). Esse tipo de inversão representou 70,3\% dos gastos brutos com atividades de PEBD em 2008, em comparação com a média europeia de 
$54,7 \%$ e a média norte-americana de $67,3 \%$ no mesmo ano. Tal índice foi superado apenas pelo Japão $(78,2 \%)$ e pela Coreia do Sul $(72,9 \%)$ (EUROSTAT EUROPEAN COMMISSION, 2008b, 2009).

Contudo, a tendência das pesquisas conduzidas por esse setor é uma concentração no desenvolvimento de inovações em matéria de produtos, devido à busca constante das empresas pelo lucro e, portanto, por projetos que ofereçam resultados imediatos. As aplicações do setor público em P\&BD, por sua vez, estão mais voltadas às inovações com impactos perceptíveis apenas em longo prazo. Mesmo assim, é o conjunto de investimentos dos setores privado e público que impulsiona a atividade de PED finlandesa e gera as condições para o aumento da competitividade nacional e para o crescimento econômico (ALI-YRKKÖ; HERMANS, 2002, p. 5; WERNER, 2003, p. 5-8).

Fontes terciárias de investimento, tais como as contribuições de instituições de ensino superior e de fundações não governamentais, mantêm-se a níveis baixos, com $0,1 \%$ e $1,1 \%$ do total de investimentos em PED, respectivamente, em 2009. As inversões externas, por sua vez, cresceram desde a crise de 1990, quando representavam 1,3\%. Em 2009, já figuravam 6,6\% do total investido, valor passível de progresso se comparado com os $10,5 \%$ suecos ou os $17,7 \%$ do Reino Unido (EUROSTAT EUROPEAN COMMISSION, 2009).

A estratégia finlandesa de criação de um SNI também alocou a população no sentido de concretizar as metas estabelecidas desde o Documento branco de 1993. Isso porque a Finlândia passou a empregar quantidade crescente de mão de obra nas atividades de PEDD. Em 2006, o total de pesquisadores representava 3,27\% da população economicamente ativa (PEA) (PEA), enquanto na União Europeia empregava-se apenas $1,54 \%$ da PEA. Do total de mão de obra utilizada no desenvolvimento de inovações tecnológicas e científicas, 56\% concentraram-se no setor privado, $31 \%$ nas instituições superiores e apenas $11 \%$ no setor público (EUROSTAT EUROPEAN COMMISSION, 2009).

Desde 1990, as políticas econômicas finlandesas coordenam-se a fim de propiciar um ambiente inovador capaz de assegurar a competitividade nacional e estabelecer uma economia do conhecimento como estratégia de desenvolvimento (WERNER, 2003, p. 12). O núcleo dessa nova economia, o setor de TIC, foi vital na geração de vantagens competitivas (DAHLMAN; ROUTTI; YLÄ-ANTTILA, 2007, p. 16; SCHIENSTOCK, 2007, p. 106-111), e isso deve-se à influência da maior empresa do país, a Nokia (BOSCHMA; SOTARAUTA, 2007, p. 164).

\section{Tecnologia da Informação e Comunicação}

O corpo conselheiro de processamento automático de dados, em 1975, e o conselho de tecnologia, em 1979, passaram a produzir relatórios destacando os 
potenciais da indústria de TIC para o crescimento do país. Tais relatórios também enfatizavam o papel da educação e dos investimentos em PẺD na germinação de um setor de TIC (BOSCHMA; SOTARAUTA, 2007, p. 165; WERNER, 2003, p. 11-12).

Ao longo da década de 1990, o conglomerado de TIC centrado no setor de equipamentos tornou-se o principal responsável pela competitividade internacional e pelo crescimento acelerado da Finlândia a níveis superiores à média europeia, especialmente entre 1997 e 2000, devido ao boom internacional de TIC (ver Gráfico 6) (ALI-YRKKÖ; HERMANS, 2002; DAHLMAN; ROUTTI; YLÄ-ANTTILA, 2007, p. 75; JALAVA, 2002; JONUNG; KIANDER; VARTIA, 2008, p. 33; KIANDER, 2004, p. 12). É importante ressaltar que o cluster finlandês de TIC abarca toda infraestrutura, produção e operação relacionadas à difusão de telecomunicações, incluindo portais e terminais disponíveis aos usuários finais, e constitui-se de cerca de 6000 empresas, entre as quais a Nokia é o núcleo.

Gráfico 6 - Especialização em TIC por país (2001)

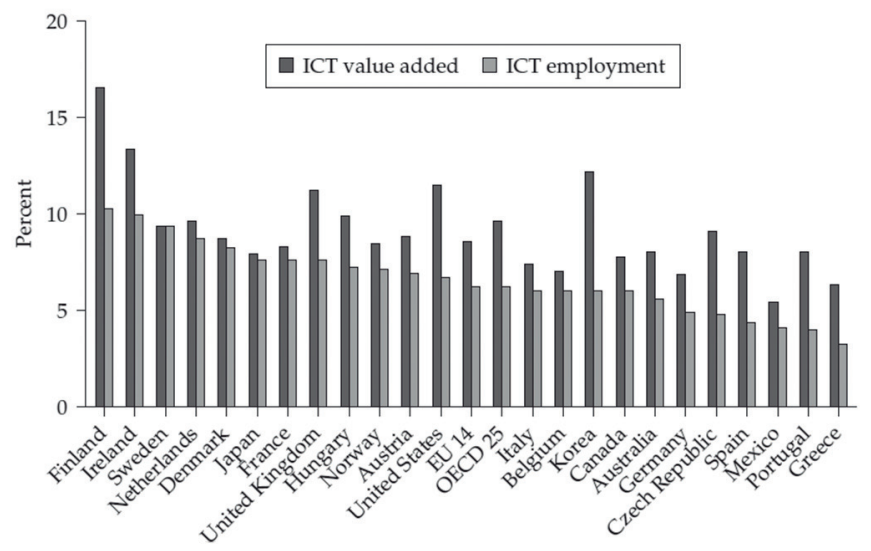

Fonte: Dahlman, Routti e Ylä-Anttila (2007).

Nesse período, o setor de TIC aumentou sua produtividade em 10 vezes, principalmente devido ao processo de destruição criativa ocasionado pela reestruturação produtiva forçada pela recessão econômica (JALAVA; HEIKKINEN; HJERPPE, 2002; KIANDER, 2004, p. 12-16; MALIRANTA; ROUVINEN; YLÄ-ANTTILA, 2010, p. 70-73). Nesse contexto, sua parcela na constituição do PIB finlandês aumentou de apenas 4\%, em 1990, para 10\%, em 2005 (DAHLMAN; ROUTTI; YLÄ-ANTTILA, 2007, p. 16). Com isso, tornou-se o terceiro pilar da economia finlandesa, junto das tradicionais indústrias florestal e metalúrgica (BOSCHMA; SOTARAUTA, 2007, p. 165). 
A participação do setor de TIC na balança externa finlandesa também aumentou desde meados dos anos 1990 e tornou-se uma parte vital na manutenção de um superávit em bens de alta tecnologia até 2009 (ver Gráfico 7) (SCHIENSTOCK, 2007, p. 106). Isso porque corresponde a mais de $80 \%$ da pauta de exportação finlandesa de produtos de alta tecnologia em comparação com a média europeia de 30\% (EUROSTAT EUROPEAN COMMISSION, 2007b, KIANDER, 2004, p. 14). A exportação de alta tecnologia que, por sua vez, correspondia a $6 \%$ das exportações em 1991, incrementou sua participação para 23\%, em 2000, e decaiu para $10 \%$, em 2010, especialmente devido à crise de 2008 e à diversificação produtiva buscada desde então (FINNISH SCIENCE AND TECHNOLOGY INFORMATION SERVICE, 2011).

Gráfico 7 - Participação da alta tecnologia no total exportado (1985-2008)

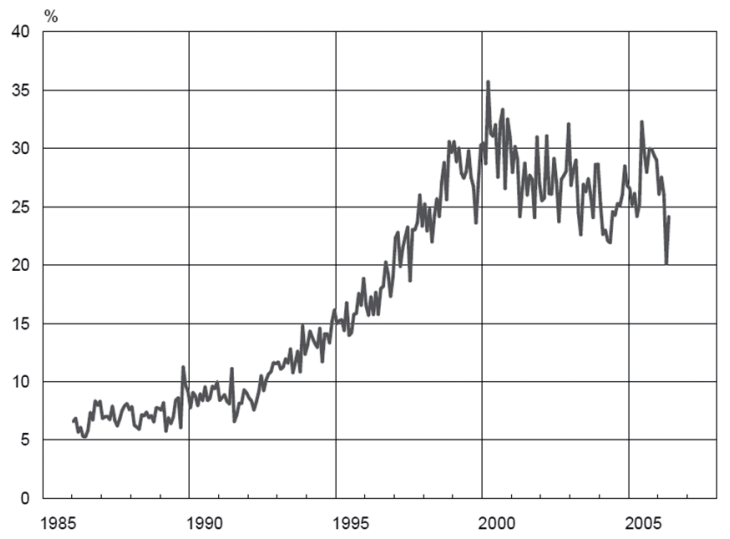

Fonte: Honkapohja et al. (2009).

A influência dos clusters de TIC na intensidade de PED nacional também é significativa, pois, enquanto em 1991 essas indústrias eram responsáveis por $17 \%$ do total investido pelo setor privado (ORGANISATION FOR ECONOMIC CO-OPERATION AND DEVELOPMENT, 2002,), em 2001 essa participação alcançou $50 \%$. Além disso, o número de pedidos de patentes - importante medida da intensidade da inovação - do setor posiciona o país na liderança mundial em intensidade de PED em TIC (EUROPEAN COMMISSION, 2003; DAHLMAN; ROUTTI; YLÄ-ANTTILA, 2007, p. 16-21, 49-52; SCHIENSTOCK, 2007, p. 107).

A aposta finlandesa nos conglomerados de TIC, bem como seu desempenho satisfatório na promoção de dinamismo e de crescimento econômico decorre de diversos fatores (SCHIENSTOCK, 2007, p. 106-275). Como primeiro entre os fatores, tem-se que as condições estruturais para a concretização dessa estratégia já haviam sido lançadas ao longo da história do país. A inexistência de monopólio 
estatal sobre o setor de telecomunicações, a abertura total do mercado à competição nacional e externa e a liberação nacional de linhas telefônicas antes da Europa continental asseguravam uma estrutura fértil à germinação de tecnologias da informação. Além disso, a aplicação de políticas que garantiam um ambiente competitivo e a proteção ao consumidor permitiu que os serviços de telefonia digital móvel oferecessem custos mais baixos e de boa qualidade, ampliando a penetrabilidade de tecnologias de telecomunicações entre a população (DAHLMAN; ROUTTI; YLÄ-ANTTILA, 2007, p. 72-74; MEINANDER, 2011, p. 141).

O segundo fator está relacionado ao fato de que, desde o pós-Segunda Guerra, o país começou a criar condições institucionais que levassem em consideração a importância das tecnologias de informação como opção produtiva viável ao contexto finlandês. Nesse sentido, diversas agências públicas foram criadas com o intuito de fornecer fundos prioritários a atividades de PEBD voltadas para o setor de TIC (DAHLMAN; ROUTTI; YLÄ-ANTTILA, 2007, p. 54).

Já o terceiro fator considera que as condições macroeconômicas internas de recessão em 1990 tornaram claro o esgotamento de um ciclo produtivo centrado exclusivamente nas indústrias florestal e metalúrgica. Com isso, aprofundaram-se as reestruturações produtivas inovadoras, entre as quais se destacou a indústria de TIC.

O quarto fator é referente ao fato de discursos favoráveis dos Estados Unidos e da União Europeia a tecnologias da informação instigaram a formulação de uma estratégia de inovação. Essa estratégia se voltava para o fomento dos condicionantes para o crescimento econômico centrado no cluster de TIC.

Tem-se ainda, como quinto fator, o fato de a década de 1990 ter testemunhado um boom do setor de TIC, tanto por causa da disponibilidade de opções de financiamento de desenvolvimentos na área, quanto por ocasião das expectativas positivas para o crescimento internacional dessa indústria. Esse ambiente externo favoreceu a reestruturação econômica em direção à produção de TICs.

Como sexto fator diz respeito à natureza do povo finlandês, que, adepto e aberto ao uso de tecnologias, constituiu um mercado consumidor exigente e qualificado. Esse mercado representou um campo de testes para futuros desenvolvimentos produtivos do cluster nascente de TIC finlandês, estabelecendo um alto nível nas exigências produtivas futuras (DAHLMAN; ROUTTI; YLÄ-ANTTILA, 2007, p. 82; INTERNATIONAL TELECOMMUNICATION UNION, 2011; OINAS, 2005, p. 1236).

Finalmente, a orientação educacional no sentido de suprir a demanda por mão de obra altamente qualificada forneceu o capital humano necessário à execução da estratégia de inovação centrada no setor de TIC. Na economia finlandesa, a conjugação desses fatores abriu espaço para o estabelecimento de uma economia do conhecimento por natureza. Com isso, a Finlândia passou a figurar posições de destaque em avaliações internacionais de desenvolvimento, de sofisticação tecno- 
lógica, de investimentos em infraestrutura e de inovação e de aproveitamento de benefícios provenientes da indústria de TIC (HONKAPOHJA et al., 2009, p. 98-99; SCHIENSTOCK, 2007, p. 95-97).

A partir de 2000, a recessão internacional e o rompimento da bolha de tecnologias da informação, que ocorreu devido à frustração das expectativas de crescimento internacional da demanda por essas tecnologias, marcaram o fim de uma fase de crescimento acelerado e o início de um crescimento mais lento (DAHLMAN; ROUTTI; YLÄ-ANTTILA, 2007, p. 19; OINAS, 2005, p. 1239). Mesmo assim, a reestruturação econômica já avançava a passos largos, e um retorno à economia natural era inadmissível. Por isso, o país ampliou o rol de políticas públicas direcionadas à profusão de inovações tecnológicas centradas no setor de TIC, a fim de assegurar a vantagem competitiva no cenário internacional (DAHLMAN; ROUTTI; YLÄ-ANTTILA, 2007, p. 21).

Em 2008, novos obstáculos provocados pela conjuntura externa de crise internacional, de difusão globalizada das TICs e de incremento da competição no setor impuseram adaptações à estratégia de inovação esboçada em 1990. Todavia, a produção de tecnologia da informação continuou impulsionando a internacionalização das firmas finlandesas, especialmente a Nokia (DAHLMAN; ROUTTI; YLÄ-ANTTILA, 2007, p. 16).

Mesmo depois das grandes mudanças internacionais e da crise, o Grupo Nokia continua exercendo um importante papel na economia finlandesa (CZARNITZKI; EBERSBERGER; FIER, 2004, p. 5; HONKAPOHJA et al., 2009, p. 105). Sua contribuição para o PIB nacional, em 2008, foi de 2,6\% e, em 2009, de 1,6\%. Todavia, as transformações estruturais do mercado internacional já não permitem que o Grupo Nokia disponha das vantagens existentes em 1990. Como consequência, em contraposição ao aumento progressivo do valor agregado da empresa, sua contribuição ao crescimento do PIB finlandês decresceu. Em termos sociais, o total de empregos criados pela empresa na economia finlandesa também revelou queda e, em 2008, chegou a apenas 0,9\% (ALI-YRKKÖ, 2010, p. 4-8).

Entretanto, a Nokia conservou seu papel de protagonista do SNI, mesmo após a diversificação de seus investimentos por 16 países (ALI-YRKKÖ, 2010, p. 20-25; OINAS, 2005, p. 1234). Na Finlândia, em 2008, seus gastos em PED alcançaram um terço do total de 3,4\% do PIB investidos nessa atividade. A empresa também foi alvo de $36,9 \%$ dos gastos públicos e responsável por $49,7 \%$ dos investimentos privados em PEBD (ALI-YRKKÖ, 2010, p. 4). Porém, houve redução relativa dos investimentos públicos nas atividades da empresa para apenas $0,16 \%$ do total gasto, em 2009, pela companhia em PED. Mesmo assim, o Grupo Nokia mantém seu dinamismo inovador, fato que se comprova com a intensidade de pedidos de patente da empresa no Organismo Europeu de Patentes. 


\section{Considerações Finais}

A trajetória inovadora da qual brotou a nova economia da Finlândia após a crise de 1990, embasada num estado de bem-estar social e em instituições estáveis, proporcionou uma transformação produtiva que deslocou a economia de seu eixo central - recursos naturais - e construiu um terceiro pilar econômico igualmente importante. Esse novo sistema produtivo de alto valor agregado e intensivo em conhecimento centrou-se nas TICs, nos investimentos em PED e em inovações como estratégia de geração de competitividade. Altos índices de competitividade são frequentemente relacionados à inserção internacional de um país e à capacidade que sua economia tem de gerar renda e bem-estar para sua população (ARBIX; FERRAZ, 2009, p. 20). Portanto, é uma medida da eficiência da trajetória desenvolvimentista adotadas por uma nação.

Na Finlândia, a estratégia nacional de inovação, formalizada apenas em 2008, promoveu aumento da produtividade e do crescimento econômico e permitiu que o país alcançasse o nível de desenvolvimento dos países centrais europeus (CAPPELEN et al., 2003, p. 4-5; KOKKINEN et al., 2007). Além disso, catapultou o país ao topo dos índices internacionais de competitividade. Nos relatórios de competitividade global do Fórum Econômico Mundial (FEM), de 2006 a 2009, a Finlândia era o sexto país mais competitivo do mundo, atrás de países como Suíça, Estados Unidos, Suécia e Dinamarca (FEM, 2006, 2007, 2008, 2009, 2010, 2011). Em 2010, o país perdeu uma posição para o Japão, mas reergueu-se para a quarta posição em 2011, além de se tornar o terceiro país mais competitivo da União Europeia (UNIÃO EUROPEIA, 2009). No ranking mundial de competitividade do International Institute for Management Development - IMD (2010, 2011), o país alcançou a 19 ${ }^{\mathrm{a}}$ posição, em 2010, e a 15ª em 2011 (FEM, 2006, 2007, 2008, 2009, 2010, 2011).

Parte desse alto patamar competitivo decorre da orientação inovadora do sistema produtivo configurado num Snif. De acordo com o Innovation Union Scoreboard de 2010 (IUS 2010) e com a Community Innovation Survey, de 2006 (CIS 2006), a Finlândia se encontra, junto da Dinamarca, Suécia e Alemanha, entre os líderes da União Europeia em desempenho inovador e na intensidade de PED (EUROPEAN COMMISSION, 2011, PARVAN, 2007). A atividade de PED recebe investimentos que representam 3,93\% do PIB finlandês, próximo da meta do bloco europeu, para 2020, de 4\% do PIB destinados a essa atividade. Parte disso decorre do aumento dos investimentos públicos em PED para 1,1\% do PIB após o enfraquecimento do setor privado, quando da recessão mundial de 2008-2009.

A estratégia nacional de inovação finlandesa, movida pela atividade de PEBD, pela liderança inovadora e pela manutenção da competitividade nacional através de um sistema de inovação, contribuiu para a redução do desemprego, o cres- 
cimento do PIB e o desenvolvimento econômico finlandês. As taxas de desemprego finlandesas declinaram lentamente desde a crise de 1990, como resultado da ampliação do setor manufatureiro provocada pelas políticas governamentais focadas na construção de uma economia do conhecimento (KIANDER, 2004, p. 14). Enquanto, durante a depressão, o desemprego nacional saltou para $20 \%$, nos últimos anos mantém-se por volta de 8\% (ORGANISATION FOR ECONOMIC CO-OPERATION AND DEVELOPMENT, 2010; STATISTICS FINLAND, 2011).

Entretanto, o impacto mais significativo da nova economia transparece no crescimento do PIB finlandês e, consequentemente, na renda per capita nacional. Isso porque a estratégia de inovação não somente reverteu o declínio do PIB em 1990, mas também produziu um crescimento que foi acelerado até 2001, alcançando 6,3\% em 1997 (ORGANISATION FOR ECONOMIC CO-OPERATION AND DEVELOPMENT, 2010; ORNSTON, REHN, 2006, p. 8). Após a crise mundial de 2008, houve retraimento do crescimento em $8,2 \%$. Porém, ainda assim, a eficiência da opção inovadora finlandesa ficou evidente através de uma rápida recuperação do crescimento após breve queda dos índices macroeconômicos, contrariando previsões negativas das autoridades econômicas (BANK OF FINLAND, 2010; WERNER, 2003, p. 16).

Enfim, o esforço integrado dos setores público e privado no fortalecimento do Snif, dos investimentos em PED e do setor de TIC criou condições para a superação de uma economia baseada em recursos naturais e para o sucesso no catching-up em relação aos países centrais europeus (CZARNITZKI; EBERSBERGER; FIER, 2004, p. 5; KIANDER, 2004, p. 23; SCHIENSTOCK, 2007, p. 104-105). Esse milagre finlandês foi produto de diversos fatores políticos, sociais e econômicos que contribuíram para o desempenho positivo da economia finlandesa após a crise de 1990 .

\section{Referências}

ACADEMY OF FINLAND. About us. 2011. Disponível em: <http://www.aka.fi/en-GB/A/Academy-of-Finland>. Acesso em: 10 jul. 2011.

ALI-YRKKÖ, J. Nokia and Finland in a sea of change. Helsinki: Taloustieto Oy; The Research Institute of the Finnish Economy, 2010.

ALI-YRKKÖ, J.; HERMANS, R. Nokia in the finnish innovation system. Helsinki: The Research Institute of the Finnish Economy, 2002. (ETLA Discussion Paper, n. 811).

ANDERSEN, E. S. et al. Schumpeter's process of creative destruction and the Scandinavian systems: a tale of two effects. In: DRUID CONFERENCE, 2006, Copenhagen. Proceedings... Copenhagen: Copenhagen Business School, 2006. p. 1 - 25.

ARBIX, G.; FERRAZ, J. V. Finlândia: competitividade e economia do conhecimento. In: CARDOSO JUNIOR, J. C.; IOLY, L. A.; MATIJASCIC, M. Trajetórias recentes de 
desenvolvimento: estudos de experiências internacionais selecionadas. v. 2. Brasília, DF: Ipea, 2009. Cap. 6. p. 221-246.

ARCHIBUGI, D.; DENNI, M.; FILIPPETTI, A. Global innovation scoreboard (eis): the dynamics of the innovative performances of countries. 2009. Bruxelas: Italian National Research Council, 2009. 51 p. (Measuring Innovation Thematic Paper).

BANK OF FINLAND. Bank of Finland Annual Report. Helsinki: Bank of Finland, 2010. 153 p.

BLOMSTRÖM, M.; KOKKO, A.; SJÖHOLM, F. Growth and innovation policies for a knowledge economy: experiences from Finland, Sweden and Singapore. Stockholm: The European Institute of Japanese Studies, 2002. 53 p. (EIJS Working Paper Series, n. 156).

BOSCHMA, R. A.; SOTARAUTA, M. Economic policy from an evolutionary perspective: the case of Finland. International Journal of Entrepreneurship And Innovation Management, v. 7, n. 2-5, p. 156-163, 2007.

CAPPELEN, A. et al. The impact of EU regional support on growth and convergence in the European Union. Journal of Common Market Studies, v. 41, n. 9, p. 621-644, 2003.

CZARNITZKI, D.; EBERSBERGER, B.; FIER, A. The relationship between RED collaboration, subsidies and patenting activity: empirical evidence from Finland and Germany. Mannheim: Centre for European Economic Research, 2004. 29 p. (Discussion Paper, n. 4-37).

DAHLMAN, C. J.; ROUTTI, J.; YLÄ-ANTTILA, P. (Ed.). Finland as a knowledge economy: elements of success and lessons learned. Washington: The World Bank, 2007. 118 p.

DAVERI, F. The new economy in Europe (1992-2001). Milão: Innocenzo Gasparini Institute For Economic Research, 2002. 36 p. (Working Paper, n. 213).

ERVASTI, H. Unemployment and unemployment policy in Finland. In: ANDERSEN, J. G. et al. (Ed.). Europe's new state of welfare: unemployment, employment policies and citizenship. Bristol: The Policy Press, 2002. Cap. 10. p. 181-193.

EUROPEAN COMMISSION. Europe's digital competitiveness report. main achievements of the Lisbon strategy 2010. Luxembourg: Publications Office of the European Union, 2009. 203 p. Disponível em: <http://ec.europa.eu/information_society/eeurope/i2010/docs/annual_report/2009/digital_competitiveness.pdf>. Acesso em: 29 ago. 2011.

Third European report on science and technology indicators. Luxembourg: Office for

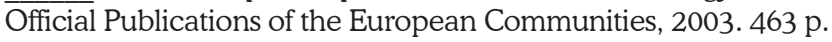

EUROSTAT EUROPEAN COMMISSION. Global Competitiveness Report 2007-2008. Genebra: WEF, 2007a. 608 p.

. Global Competitiveness Report 2008-2009. Genebra: WEF, 2008a. 513 p.

. Global Competitiveness Report 2009-2010. Genebra: WEF, 2009. 492 p.

. Global Competitiveness Report 2010-2011. Genebra: WEF, 2010. 516 p.

. Global Competitiveness Report 2011-2012. Genebra: WEF, 2011. 544 p. 
Science, technology and innovation in Europe. 2007. Luxembourg: Office for Official Publications of the European Communities, 2007b. 156 p. (Eurostat Pocketbooks).

. Science, technology and innovation in Europe. 2008. Luxembourg: Office for Official Publications of the European Communities, 2008b. 244 p. (Eurostat Statistical Books).

FINLAND. Ministério do Comércio e da Indústria (atual Ministério do Emprego e da Economia). Finnish national industrial strategy white paper. Helsinki: Ministério do Comércio e da Indústria, Departamento da Indústria, 1993. 143 p.

. Ministério do Emprego e da Economia. Prime Minister Matti Vanhanen's Second Cabinet's Government Programme. Government's Communication on Finland's National Innovation Strategy to the Parliament. Helsinki, 2008. 42 p.

. Ministry of Education and Culture. Research and innovation council. Disponível em: $\overline{<\mathrm{http}}$ //www.minedu.fi/OPM/Tiede/tutkimus-_ja_innovaationeuvosto/?lang=en $>$. Acesso em: 20 jul. 2011.

. Ministry of Employment and the Economy. Strategic centres and clusters. Disponível:

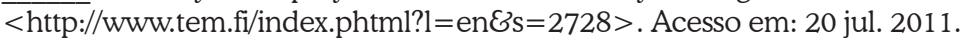

FINNISH SCIENCE AND TECHNOLOGY INFORMATION SERVICE. Performance. 2011. Disponível em: < http://www.research.fi/en/performance >. Acesso em 13 ago. 2011.

FURMAN, J. L.; PORTER, M. E.; STERN, S. The determinants of national innovative capacity. Research Policy, v. 31, p. 899-933, 2002.

GRÖNQVIST, C.; KINNUNEN, H. Impact of recession on labour supply: experiences from the 1990s in Finland. 2. ed. Helsinki: Bank of Finland, 2009. 18 p.

HONKAPOHJA, S. et al. Economic prosperity recaptured: the finnish path from crisis to rapid growth. Cambridge; Londres: Massachusetts Institute of Technology Press, 2009. 145 p.

HONKAPOHJA, S.; KOSKELA, E. The economic crisis of the 1990s in Finland. Helsinki: The Research Institute of the Finnish Economy, 1999. 78 p.

HONKAPOHJA, S.; KOSKELA, E.; PAUNIO, J. The great depression of the 1990s in Finland. Finnish Economic Papers, Helsinki, v. 9, n. 1, p. 37-54, 1996.

INTERNATIONAL INSTITUTE FOR MANAGEMENT DEVELOPMENT. World Competitiveness Yearbook. Lausanne, Switzerland: IMD World Competitiveness Center, 2010. Disponível em: < http http://www.worldcompetitiveness.com/OnLine/App/Index.htm Acesso em: 2 ago. 2011.

. World Competitiveness Yearbook. Lausanne, Switzerland: IMD World Competitiveness Center, 2011. Disponível em: < http://www.worldcompetitiveness.com/OnLine/App/Index. htm >. Acesso em: 2 ago. 2011.

JALAVA, J. The production and use of ICT in Finland. Helsinki: The Research Institute of the Finnish Economy, 2002. 31 p. (ETLA Discussion Paper, n. 827). 
JALAVA, J.; HEIKKINEN, S.; HJERPPE, R. Technology and structural change: productivity in the Finnish manufacturing industries, 1925-2000. Varsóvia: Tiger, 2002. 21 p.

JAUHIAINEN, J. Regionl and Innovation Policies in Finland: Towards Convergence and/or Mismatch? Regional Studies, Seaford, v. 42, n. 7, p. 1031-1045, 2009.

JONUNG, L.; KIANDER, J.; VARTIA, P. The great financial crisis in Finland and Sweden: the dynamics of boom, bust and recovery, 1985-2000. Brussels: European Commission, Directorate-general for Economic and Financial Affairs, 2008. 72 p. (Economic Papers, 350).

JONUNG, L.; SÖDERSTRÖM, H. T.; STYMNE, J. Depression in the North: boom and bust in Sweden and Finland, 1985-1993. Finnish Economic Papers, Helsinki, v. 9, n. 1, p. 55-71, 1996.

KAUTTO-KOIVULA, K. et al. Leading the way to new creative knowledge age. Helsinki: Global University Sistem, Feb. 2008. 22 p. (Paradigm Shift Project).

KIANDER, J. The evolution of the finnish model in the 1990s: from depression to high-tech boom. Helsinki: Government Institute for Economic Research, 2004. 39 p.

KIANDER, J.; VARTIA, P. The great depression of the 1990s in Finland. Finnish Economic Papers, Helsinki, v. 9, n. 1, p. 72-88, 1996.

KIANDER, J.; WIDGRÉN, M. Introduction: The Finnish Economic Crisis of the 1990s. Finnish Economic Papers, Helsinki, v. 9, n. 1, p. 3-5, 1996.

KIRBY, D. A concise history of Finland. 4. ed. Cambridge, Nova Iorque, Melbourne, Madri, Cidade do Cabo, Cingapura, São Paulo, Nova Delhi, Dubai, Tóquio: Cambridge University Press, 2009. 345 p.

KLINGE, M. Breve história da Finlândia. 3. ed. Tradução de Wamberto Hudson Ferreira e Carlos Eduardo Guedes. Helsinki: Otava, 2000. 176 p.

KOKKINEN, A. et al. Catching up in Europe: Finland. Helsinki: Pallervo Economic Research Institute, 2007. 31 p. (Working Papers, n. 93).

MALIRANTA, M.; ROUVINEN, P.; YLÄ-ANTTILA, P. Finland's path to the global productivity frontier through creative destruction. International Productivity Monitor, Ottawa, n. 20, p. 68-84, 2010.

MEINANDER, H. A history of Finland. London: C. Hurst E Company, 2011. 227 p.

OINAS, P. Finland: a success story? European Planning Studies, v. 13, n. 8, p. 1227-1244, Dec. 2005.

ORGANISATION FOR ECONOMIC CO-OPERATION AND DEVELOPMENT. Country statistical profiles: key tables from OCDE. Paris: OECD Publishings, 2010. Disponível em: <http:// dx.doi.org/10.1787/20752288-2010-table-fin > . Acesso em: 28 set. 2011.

. Science, technology and industry outlook 2002. Paris: OECD Pulishings, 2002. 263 p.

Study on digital learning resources as systemic innovation: country case study report on Finland. Paris: OECD Pulishings 2008. p. 22. 
ORNSTON, D.; REHN, O. An old consensus in the 'New' Economy? Institutional adaptation, technological innovation and economic restructuring in Finland. In: ZYSMAN, J.; NEWMAN, A. (Ed.). How revolutionary was the digital revolution? Stanford: Stanford University Press, 2006. Cap. 4.

PALMBERG, C. et al. Industrial innovation in Finland: first results of the Sfinno-project. Espoo: Vtt, Group For Technology Studies, 2000. 87 p. (Working Paper, n. 47).

PARLIAMENT OF FINLAND. Committee for the Future. Disponivel em: < http://web.eduskunta.fi/Resource.phx/parliament/committees/future.htx>. Acesso em: 29 jul. 2011.

PARVAN, S. Community Innovation Statistics: Fourth Community Innovation Survey (CIS 4) and European Innovation Scoreboard (EIS) 2006. Statistics In Focus: Science and Technology, Luxembourg, n. 116, p.1-8, 2007.

POWELL, W.; SNELLMAN, K. The knowledge economy. Annual Review of Sociology, v. 30, p. 199-220, Feb. 2011.

SCHIENSTOCK, G. From path dependency to path creation: Finland as a case in point. Current Sociology, v. 55, n. 1, p. 92-109, Jan. 2007.

SINGLETON, F. A short history of Finland. 2. ed. Cambridge, Nova Iorque, Melbourne, Madri, Cidade do Cabo, Cingapura, São Paulo, Nova Delhi, Dubai, Tóquio: Cambridge University Press, 1998. 209 p.

SITRA. About Sitra. Disponível em: <http://www.sitra.fi/en/about-sitra> . Acesso em: 11 jul. 2011.

STATISTICS FINLAND. Unemployment rate 6.9 per cent in September. 25 Oct. 2011. Disponível em: <http://www.stat.fi/til/tyti/2011/09/tyti_2011_09_2011-10-25_tie_001_en.html>. Acesso em: 29 jul. 2011.

STUDY IN FINLAND. Where to study? Higher education institutions in Finland. Disponível em: <http://www.studyinfinland.fi/institutions >. Acesso em: 20 jul. 2011.

TEKES. About us. Disponível em: < http://www.tekes.fi/en/community/About_Tekes/339/ About_Tekes/1279>. Acesso em: 10 jul. 2011.

TOLEDO, D. G. C.; ARBIX, G. A. T.; SALERNO, M. S. Estratégias de inovação em sete países: Estados Unidos, Canadá, Irlanda, Reino Unido, Finlândia, França e Japão. In: ENCONTRO ANUAL DA ANPOCS, 33., 2009, Caxambu. Relatório Final. Caxambu: Anpocs, 2009. p. 1-25.

UUSITALO, H. Economic crisis and social policy in Finland in the 1990s. Sidney: Social Policy Research Centre, 1996. 39 p. (SPRC Discussion Papers, n.70).

VERHO, J. Scars of recession: the long-term costs of the finnish economic crisis. Uppsala: Institute for Labour Market Policy Evaluation, 2008. 38 p. (Working Paper, n. 9).

WERNER, R. Finland: a European model of successful innovation. Chazen Web Journal of International Business, p. 1-19, Fall 2003. 
YLÄ-ANTTILA, P.; LEMOLA, T. Transformation of innovation system in a small country: the case of Finland. In: CONFERÊNCIA GLOBELICS, 2003, Rio de Janeiro. Anais eletrônicos... Helsinki: organizador? 2003. 21 p. Disponível em: <www.advansis.fi/docs/globelics_text. doc >. Acesso em: Acesso em: 4 jul. 2011.

Recebido em: 06/02/2012. Aceito em: 30/05/2014. 